\title{
Modified 0-1 Knapsack Problem for Demand Side Management
}

\author{
Siddarth Sankar M J, Rahul Sharma K
}

\begin{abstract}
Demand Side Management plays an important role in the maintenance of Smart Grid stability and allows to optimally manage consumer energy consumption. The advantages of Demand Side Management are improved energy efficiency and stability, reduction of electricity production cost and improved grid reliability. The various techniques of Demand Side Management are Direct Load Control, Smart Pricing, Demand Bidding, etc. Evolutionary algorithms and linear programming approach used for Demand Side Management considers cost function-based optimization for Direct Load Control. This paper proposes a Direct Load Control technique using a modified 0-1 Knapsack Problem. This technique alters the load during the peak time when demand is more, based on the consumer's demand. Furthermore, backup load management is proposed, which will be used when demand is more during peak load time based on the total available supply. If backup load usage exceeds the set limit, load will be cut off and the period of cut off will be based on the Fibonacci timing period. The proposed algorithms are simulated in MATLAB Simulink environment and the simulation results show the effectiveness of approach.
\end{abstract}

Index - Smart Grid, Demand Side Management, Demand Response, Direct Load Control, 0-1 Knapsack Problem, Backup load management.

\section{INTRODUCTION}

Smart Grid is the futureprospect of electrical grid. It is the advancement of grid with smart metering devices. And, with radical sensing and measuring of power by integration of two-way communication using information technology. The major objectives of Smart Grid are improved energy efficiency, emission reduction by usage of the renewable energy sources generated at the consumer side [1], etc.

The concept of distributed generation in Smart Grid is sophisticated to maintain the stability. A method to improve grid stability, Demand Side Management (DSM) is used in [2]. The important aspect of DSM is the balance of load and supply in the grid. [1].

In developing countries, the electrical grid infrastructure is in the development stage where the advanced metering and sensing units are yet to be established. DSM plays a vital role in this context by reducing energy consumption and improving stability of the system. DSM techniques influence the consumers to reduce the demand during peak hours [3][4].

The various techniques adopted for DSM are Direct Load Control (DLC), load limiters, commercial/industrial programs, frequency regulation, Smart Pricing, Demand

Revised Manuscript Received on April 12, 2019.

Siddarth Sankar M J, Department of Electrical and Electronics

Engineering, Amrita School of Engineering, Coimbatore.Amrita Vishwa Vidyapeetham, India.(E-mail: siddarthsankarz@gmail.com.)

Rahul Sharma K, Department of Electrical and Electronics Engineering, Amrita School of Engineering, Coimbatore.Amrita Vishwa Vidyapeetham, India.(E-mail: k_rahulsharma@cb.amrita.edu.)
Bidding, etc. These techniques are mainly used to control the demand side of electrical grid. In DLC based DSM technique, the loads are controlled and modified by connecting and reconnecting the loads using circuit breakers based on demand at demand side[6]. There must be a mutual agreement between the consumer and generation utility on controlling the household loads remotely when the demand is high during peak hours [4]. Several algorithms are developed for DLC over the years. An interactive evolutionary algorithm is one of them, it is a multi-objective optimization problem which considers various factors, based on decision maker's preference [5] and operations are carried outbased on Genetic operators. A profit-based load management using DLC is proposed, where the linear programming approachis used. It considers cost function and demands as major decision-making parameters. Afore mentioned algorithm considers incentive or cost function as main objective to reduce the demand.

0-1 Knapsack problem is a dynamic programming approach used for DLC. It is a combinatorial optimization method which is generally used for resource allocation problems like financial constraints, cryptography, etc. The dynamic programming approach is proposed in this paper. The consumer assigns priority to the loads [8] and the higher priority load will be included in the system during peak hours of operation [7]. The fractional knapsack algorithm is used in [10], where it considers how much consumption time is required for keeping total cost unchanged. However, a combined load management and backup load management approach is missing in literature, to best of our knowledge. Backup loads are important to the consumers especially during power outages Knapsack problem based optimization algorithm controls the loads during peak hours to minimize the demand-supply mismatch. A modified 0-1 Knapsack problem proposed in this paper proves to be more accurate for implementing in the electrical grid at the demand side. The modified 0-1 Knapsack problem applied in the central system of the electrical grid will select the loads based on the priority given by the consumer. It cuts off the whole individual household load when consumer demand exceeds a certain limit.

In the proposed backup load management strategy, an individual household consumer can use the maximum backup load capacity prescribed by the utility. If the consumption is higher than the prescribed capacity, back up loads will be cut off for a period. The key question in this strategy is "when should the utility check whether the

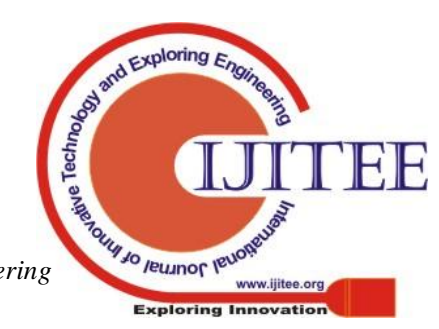


household has reduced the consumption to the prescribed capacity". A Fibonacci sequence based scheme for checking the consumption is promising, as the response time for household to react upon the situation varies in practical sense. Furthermore, the Fibonacci series based approach also avoids frequent switching at regular interval of time and will help the consumer to reduce the load if need to use backup load allotted. The added advantages of the proposed backup load management system are the flexibility in load selection (user perspective) and scalability in adding/removing the households into backup load management program (utility perspective). This strategy differs from the common demand dispatch problem where the utility control the load and the role of users is minimal; this approach allows the consumers to choose the backup loads.

The paper is organized into different sections. Section II gives overview of the proposed system. Section III describes the modified 0-1 Knapsack problem. Simulation results are shown in section IV with conclusion in section V.

\section{SYSTEM OVERVIEW}

A case study with three households are shown in Fig 1. The study consists of a micro grid along with three subsystems; power measurement unit, Load Dispatch Centre (LDC) and scopes. The micro grid consists of three phase source, three phase transformers and a linear transformer. The household units consist of normal loads and backup loads which are connected to thegrid using circuit breakers and will be controlled through a LDC.

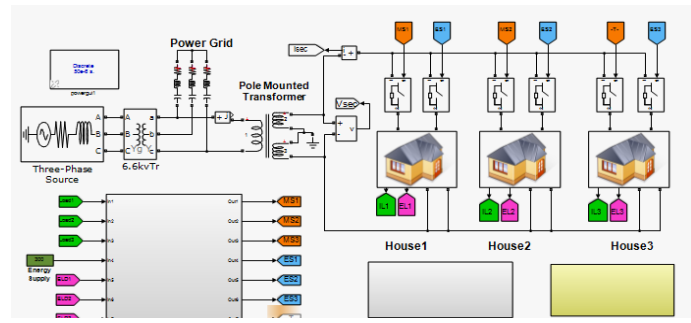

Fig. 1. A case study with three households

The power measurement unit subsystem measures power from households, backup loads and grid.The modified 0-1 Knapsack problem algorithm is implemented at the LDC. LDC subsystem runs the modified 0-1 Knapsack problem and backup load management algorithms based on the inputs from power measurement unit and the customer preference. The inputs from power measurement unit are collected by monitoring the household normal power consumption ( $\mathrm{NL}_{1}, \mathrm{NL}_{2}$ and $\mathrm{NL}_{3}$ ), backup load consumption $\left(\mathrm{BL}_{1}, \mathrm{BL}_{2}\right.$ and $\left.\mathrm{BL}_{3}\right)$ and total available power from utility. The outputs of knapsack problem and backup load management are used for switching off circuit breakers $\left(\mathrm{MS}_{1}, \mathrm{MS}_{2}\right.$, and $\left.\mathrm{MS}_{3}\right)$ for household loads and for backup loads $\left(\mathrm{ES}_{1}, \mathrm{ES}_{2}\right.$, and $\left.\mathrm{ES}_{3}\right)$. Power consumption of normal loads and back up loads will be monitored by scopes subsystem and LDC will give the required control signals.

0-1 Knapsack Problem using a Dynamic Programming approach

0-1 Knapsack problem with a dynamic programming technique is considered for the application. It is one of the

approaches which gives the best possible dominant value tocorresponding amount as the result of the algorithm [7].

Traditional 0-1 Knapsack problem using dynamic approach can be expressed (1) [9]:

$$
\max \left\{\sum_{k=1}^{n} p_{k} x_{k} \mid \sum_{k=1}^{n} w_{k} x_{k} \leq C, x_{k} \in\{0,1\}\right\}
$$

where

$C$ is capacity of the knapsack,

$N$ is number of items,

$p_{k}$ is value associated with $k$-th item,

$w_{k}$ is amount associated with $k$-th item.

Without loss of generality, all the data are assumed to be positive integers. In order to avoid trivial solutions. Assume $\sum_{k=1}^{n} w_{k}>C, w_{k}<C$ for all $k \in\{1 \ldots, n\}$.

For the proposed model $C$ is the total available generated power, $n$ is the number of loads present in the system, $p_{k}$ is each household loads power consumptions and $w_{k}$ is the priority assigned to the loads by the consumer.

Dynamic programming approach gives importance to dominant values, so that higher priorty loads can be included during high demand. The limitation of $0-1$ Knapsack problem using a dynamic programming approach for this application, is that it will not check whether higher priority load is consuming most of the generated power. So to overcome this limitation, a new set of dominant values are calculated [10].

A Modified 0-1 Knapsack problem is proposed for DSM. It is 0-1 Knapsack problem witha new set of dominant values. In Modified 0-1 Knapsack problemnew set of dominant valuesare calculated based on the power consumped by the $k^{\text {th }}$ load and available generated power from utility. The dominant values are calculated using (2).

$$
v_{k}=\left\lceil\left(p_{k} *\left\lceil 1-\frac{L_{k}}{T L}\right\rceil\right)\right\rceil
$$

where, $p_{k}$ is the priorities given for $k^{\text {th }}$ loads, $L_{k}$ is the power consumed by the $k^{\text {th }}$ load and $T L$ is the total generated power. The set ofcalculated dominant values based on priority are assigned as new set values to the 0-1 Knapsack problem. Best dominant values which satisfy (1) will be selected. The selected loads will be present in the grid, others will be cut off from grid using circuit breakers.

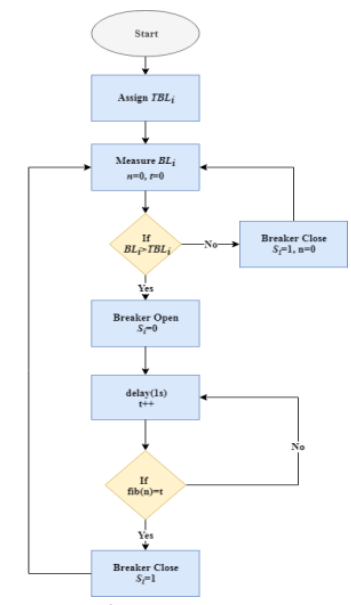

Fig.3 Flowchart of Backup Load Management 
Backup load management system is proposed to avoid power outage during peak hours. Backup load mangement will have a separate line of supplyto each consumer and it will be activated during peak hours of high demand. Theconcept ofbackup load management is shown in Fig. 3. The system will be allotted with a certain percentage of generated power from utiltiy. Based on the number of consumers, the alloted power is split equally and distributed to each load $\left(T B L_{i}\right)$ as backup load. If the power consumption for $\mathrm{i}^{\text {th }}$ backup load $\left(B L_{i}\right)$ is more than the alloted backup power for $\mathrm{i}^{\text {th }}\left(T B L_{i}\right)$, the breaker will be open for it ${ }^{\text {th }}$ backup load $\left(S_{i}=0\right)$, if it doesn't exceed, the breaker will be in closed state $\left(S_{i}=1\right)$ and set $n=0$. When breaker is in open state it will initate a delay timer for one second. When the delay timer of $i^{\text {th }}$ backup load is equal to $n^{\text {th }}$ instant of Fibonacci series (fib(n)), breaker gets closed $\left(S_{i}=1\right)$ and goes back to measure power of $\mathrm{i}^{\text {th }}$ backup load. This process continues during peak hours of high demand The fibonicci series-based approach is introdcued to the system ,suppose if consumer wants to use the backup loads with reduced consumption of power. So, a swtiching sequence is introduced for the backup load circuit breakers. Simple solution is to have switching at constant time intervel. But it may cause frequent switching of circuit breakers. So, the Fibonacci series-based approach avoids frequent switching at periodic intervals of time and will help the consumer to reduce the load, to usethe backup load allotted, if needed. Fibonacci series of $\mathrm{n}^{\text {th }}$ instant can be obtained using the algorithm shown below:

START

procedure fib( $n)$

declare: f0, fl, fib, loop, $n$

set fo to 0

set fl to 1

for loop $\leftarrow 1$ to $n$

$f i b \leftarrow f 0+f 1$

$f 0 \leftarrow f 1$

$f 1 \leftarrow f i b$

$f i b(n)=f i b$

END

end for

the $\mathrm{n}^{\text {th }}$ number of Fibonacci series is calculated and compared with time taken after opening of breaker. When it becomes equal, the breaker is closed again and timer resets and the process is repeated.

\section{SIMULATION RESULTS}

The simulation results can be discussed as two parts: Fig.4 shows the modified 0-1 Knapsack problem output for load control during high demand and Fig.5 shows backup load management for emergency loads during power outages.

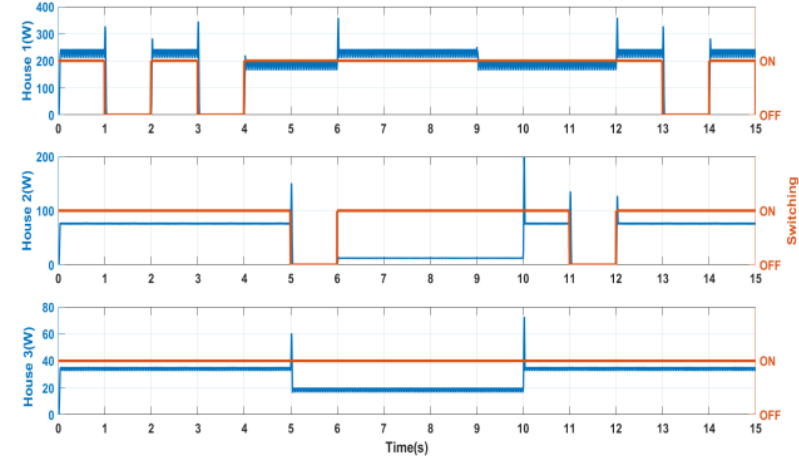

Fig. 4.Modified 0-1 Knapsack problem simulation results.

The simulation results obtained from the modified 0-1 Knapsack problem with three different house loads is shown in the Fig. 4. The algorithm will check the condition at frequency of $1 \mathrm{~Hz}$. The simulation is obtained by assuming the total available supply as 300 units and with three house consumption of power House 1, House 2 and House 3 . House 1 has the highest priority, House 2 has the lowest and House 3 has the second highest priority. Consider first scenario where House 1 is consuming around 250 units which consumes the highest percentage of available generated power. Even though House 1 is of higher priority, the algorithm will cut off the load after one second, since it is consuming utmost of generated power. At $t=2 s$, the consumption of House 1 power is unchanged. So, it will remain cut off from the supply. At $t=4 \mathrm{~s}$, House 1 decreases to around 200 units and other loads are around 75 units and 35 units. House 1 breaker closes at $t=5 \mathrm{~s}$ the load value remains unchanged. House 2 breaker cuts off during $\mathrm{t}=5 \mathrm{~s}$ to $\mathrm{t}=6 \mathrm{~s}$, since it is the lowest priority load. At $\mathrm{t}=6 \mathrm{~s}$, House 1 regains to 250 units, House 2 reduces to 10 units and House 3 consumes around 15 units. Since the overall load consumption of houses is around 275 units which meets the available generated power. So, all the breakers are in closed condition. So, the simulation results shows that the modified 0-1 Knapsack problem manages the loads efficiently.

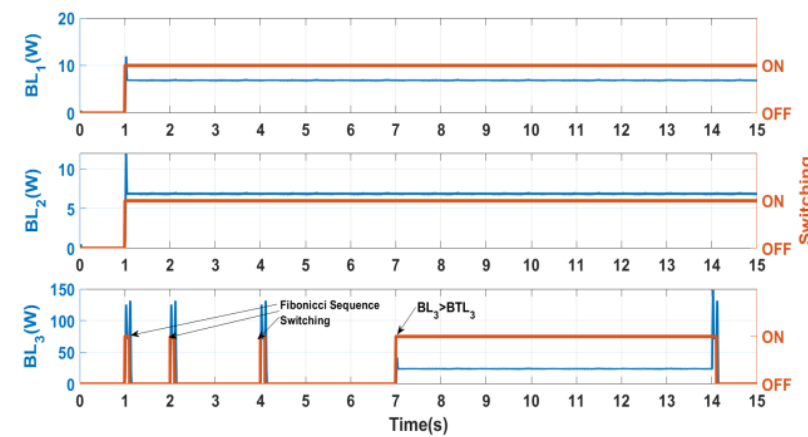

Fig. 5.Backup load management simulation results.

Backup load management simulation output is shown in Fig. 5. The system will have a separate set of breakers to control the backup load for each of the three house loads.

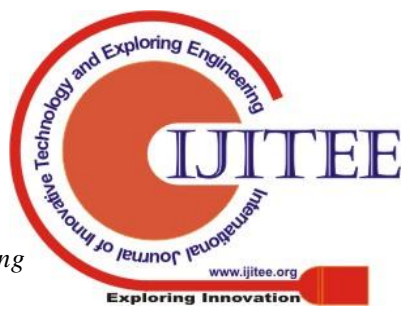


All the backup load power is measured after $t=1 \mathrm{~s}$. So, all the breakers are in open state for one second duration. After one second, breakers are closed for all backup loads $\left(\mathrm{BL}_{1}, \mathrm{BL}_{2}\right.$ and $\left.\mathrm{BL}_{3}\right)$. The power measured at every instant of time. The power consumption of $\mathrm{BL}_{1}$ and $\mathrm{BL}_{2}$ are within the limit, so breakers are in closed state. But the $\mathrm{BL}_{3}$ consumes more power than allotted load $\left(\mathrm{BL}_{3}>\mathrm{BTL}_{3}\right)$. So, the breaker of $\mathrm{BL}_{3}$ will be open and it will initiate a timer delay of one second running until it reaches the second instant of Fibonacci series (i.e. $f i b(2)=1$ ). So, the breaker will be closed at $t=2 s$, again it measures the power, but the power consumption remains unchanged. The Fibonacci series instant value is increased by one whenever the breakers opens due to increased power consumption. So, the breaker of $\mathrm{BL}_{3}$ will be in open state for two seconds (i.e. $f i b(3)=2$ ). Again, it checks the condition in $t=4 \mathrm{~s}$, still the power consumption exceeds and hence load remains cut off until $t=7 \mathrm{~s}$ (i.e. $f i b(4)=3$ ). After that at $t=7 \mathrm{~s}$ the load is reduced to less than or equal to allotted power. So, the breaker will be closed. At $t=14 \mathrm{~s}$ consumer tries to consume more power from backup supply. So, it will be cut off again.

The results above shows that proposed modified 0-1 Knapsack problem provides utility to cut off higher priority loads when it consumes more than a percentage of generated power. This feature in the algorithm makes the system to be more dependable during high demand. And backup load management system provides more flexibility and scalability to consumers and utility.

\section{CONCLUSION}

Demand Side Management helps the utility to manage the smart grid in efficient and reliable manner. It helps to optimize the under-utilized grid in terms of generation of electricity, transmission lines and distribution network. One of the DSM techniques is DLC, where the loads are controlled and modified by connecting and reconnecting the loads using circuit breakers, at demand side, based on demand. Several algorithms are developed for DLC over the years. In this paper, a modified 0-1 Knapsack problem for DLC is proposed for DSM.The loads are assigned priority by the consumers as per mutual agreement. The loads are cut off based on priority as well as the percentage of power consumption of the generated power during high demand. So, the power consumption of each loads is contained within the generated power. An additional system is proposed to give a backup load during peak time to avoid power outage. The backup load will be alloted based onthe generated power. The load is controlled as per usage of alloted power. If it exceeds, the breaker will cut off the supply for the load for a duration of time. The duration of time is decided based on the Fibonacci series. The system proposed in this paper here is flexible in load selection (user perspective) and scalable in adding/removing the households (utility perspective). This strategy differs from the common demand dispatch problem where the utility control the load and the role of users is minimal. This approach allows the consumers to choose the backup loads. The simulation results obtained from MATLAB Simulink shows the effectiveness of proposed system. The future improvements can be done for controlling individual loads which leads to a distributed optimization approach.

\section{REFERENCES}

1. T. Logenthiran, D. Srinivasan and T. Z. Shun, "Demand Side Management in Smart Grid Using Heuristic Optimization," in IEEE Transactions on Smart Grid, vol. 3, no. 3, pp. 12441252

2. Cappers, Peter \& Goldman, Charles \& Kathan, David. "Demand response in U.S. electricity markets: Empirical evidence. Energy". 35. 2009, pp.1526-1535.

3. K. Gaur, H. Kumar, R. P. K. Agarwal, K. V. S. Baba and S. K. Soonee, "Analysing the electricity demand pattern," 2016 National Power Systems Conference (NPSC), Bhubaneswar, 2016, pp. 1-6.

4. J. S. Vardakas, N. Zorba and C. V. Verikoukis, "A Survey on Demand Response Programs in Smart Grids: Pricing Methods and Optimization Algorithms," in IEEE Communications Surveys \& Tutorials, vol. 17, no. 1,2015, pp. 152-178.

5. A. Gomes, C. H. Antunes and A. G. Martins, "A Multiple Objective Approach to Direct Load Control Using an Interactive Evolutionary Algorithm," in IEEE Transactions on Power Systems, vol. 22, no. 3, Aug. 2007, pp. 1004-1011.

6. Nithin S., Sivraj P., Sasi K. K. and R. Lagerstöm, "Development of a Real Time Data Collection Unit for distribution network in a smart grid environment," 2014 POWER AND ENERGY SYSTEMS: TOWARDS SUSTAINABLE ENERGY, Bangalore, 2014, pp. 1-5.

7. P. Toth, "Dynamic programming algorithms for the Zero-One Knapsack Problem," Computing, vol. 25, no. 1, 1980, pp. 2945.

8. S. Choi, S. Park, and H.-M. Kim, "The Application of the 0-1 Knapsack Problem to the Load-Shedding Problem in Microgrid Operation," Communications in Computer and Information Science Control and Automation, and Energy System Engineering, pp. 227-234, 2011.

9. M. E. D. El Baz, "Load balancing in a parallel dynamic programming multi-method applied to the 0-1 knapsack problem," 14th Euromicro International Conference on Parallel, Distributed, and Network-Based Processing (PDP'06), Montbeliard-Sochaux, France, 2006, pp. 6.

10. O. A. Sianaki, O. Hussain and A. R. Tabesh, "A Knapsack problem approach for achieving efficient energy consumption in smart grid for endusers' life style," 2010 IEEE Conference on Innovative Technologies for an Efficient and Reliable Electricity Supply, Waltham, MA, 2010, pp. 159-164.

11. C. Yokita, S. Nithin and A. S. Pillai, "Development of a Battery Monitoring and Control Unit Aiding Utilities in Demand Side Management," 2018 International Conference on Inventive Research in Computing Applications (ICIRCA), Coimbatore, 2018, pp. 1325-1329. 